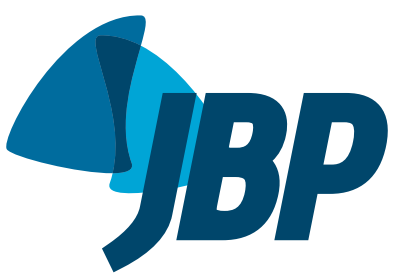

\section{PRACTICAL SCENARIO}

A meta-analysis examined the effect of the use of daily medium-dose inhaled corticosteroids (ICS) on preventing exacerbations among preschoolers with recurrent wheeze. It summarized the results of 15 randomized clinical trials (RCTs) involving 3,278 individuals that showed that the use of daily ICS, compared with that of placebo, prevented exacerbations by $30 \%$ [risk ratio $(\mathrm{RR})=0.70 ; 95 \% \mathrm{CI}$ : 0.61-0.79; number needed to treat $(\mathrm{NNT})=9$. $^{(1}$

\section{COMPARING RISKS}

The impact of interventions can be estimated by comparing the incidence of the outcome (e.g., exacerbations) in the experimental group vs. a control group (e.g., placebo) by calculating an outcome ratio or difference across the intervention groups. The typical ratio calculated is the risk in the intervention group over the risk in the control group, designated the risk ratio (RR). In RCTs, the difference in risk between groups is called the absolute risk reduction (ARR), and it represents the proportion of outcomes reduced by the new intervention to the comparison group. Similar estimates can be calculated in observational studies replacing an intervention with the exposure of interest; for example, tobacco smokers compared with nonsmokers when reporting the risk of tobacco-related disease.

A statistic related to the ARR is the NNT, which is important because it provides an estimate of the number of patients that are required to be treated to avoid one additional patient from developing the outcome of interest (Table 1). ${ }^{(2)}$

The popularity of NNT has increased considerably, although this statistic is not necessarily easier to grasp than the ARR, either by patients or physicians. It is useful to remember that the lower the NNT, the higher the effectiveness of the intervention. In our example, an NNT of 9 is interpreted as follows: 9 children, on average, need to be treated with ICS to prevent 1 additional child from having an exacerbation.

More recently, RCTs also evaluate the impact of adverse events of an intervention by reporting the number needed to harm (NNH) in addition to the NNT. NNH is defined as the average number of individuals that would need to be exposed to a new intervention to produce one additional adverse outcome.

\section{LIMITATIONS AND KEY POINTS}

The reporting of NNT and NNH should always include confidence intervals and not only a point estimate.

The importance of taking into account the baseline risk to properly assess an intervention in an RCT cannot be overemphasized. Table 1 shows that as the incidence of the outcome in the control group increases, an identical RR results in greater ARRs and, consequently, lower NNT. Therefore, the effect can be exaggerated by simply reporting an RR of 0.75 . In both examples, the risk is reduced by $25 \%$, but NNT informs how many individuals must be treated in order to decrease that risk or absolute difference. It is recommended reporting both absolute and relative effect sizes.

The size and clinical impact of the effect of the intervention are important. Similar RCTs may have the same NNT, but their clinical relevance is different if the NNT refers to preventing one death or one COPD exacerbation when compared with preventing a small decrease in $\mathrm{FEV}_{1}$ or another surrogate outcome.

Table 1. Comparing risks and interpreting results across different clinical scenarios. RR: risk ratio; ARR: absolute risk reduction; and NNT: number needed to treat. ${ }^{(2)}$

\begin{tabular}{|c|c|c|}
\hline & Result & $\begin{array}{c}\text { Interpretation } \\
\end{array}$ \\
\hline \multicolumn{3}{|c|}{$\begin{array}{l}\text { Low baseline risk (20\% risk of death in the control group) } \\
\text { Control group: } n=500 ; 100(20 \%) \text { deaths; Intervention group: } n=500 ; 75(15 \%) \text { deaths }\end{array}$} \\
\hline RR & $15 \% / 20 \%=0.75$ & The intervention reduces risk by $25 \%$ \\
\hline ARR & $20 \%-15 \%=5 \%$ & The intervention reduces risk in $5 \%$ \\
\hline NNT & $1 / 5 \%=20$ & 20 patients need to receive the intervention to prevent 1 death \\
\hline \multicolumn{3}{|c|}{$\begin{array}{l}\text { High baseline risk (50\% risk of death in the control group) } \\
\text { Control group: } n=500 ; 250(50 \%) \text { deaths; Intervention group: } n=500 ; 188(38 \%) \text { deaths }\end{array}$} \\
\hline RR & $38 \% / 50 \%=0.75$ & The intervention reduces risk by $25 \%$ \\
\hline ARR & $50 \%-38 \%=12 \%$ & The intervention reduces risk in $12 \%$ \\
\hline NNT & $1 / 12 \%=8$ & 8 patients need to receive the intervention to prevent 1 death \\
\hline
\end{tabular}

\title{
REFERENCES
}

Kaiser SV, Huynh T, Bacharier LB, Rosenthal JL, Bakel LA, Parkin PC, et al. Preventing Exacerbations in Preschoolers with Recurrent Wheeze: A Meta-analysis. Pediatrics. 2016;137(6). pii: e20154496. https://doi.org/10.1542/peds.2015-4496
Laupacis A Sackett DL Roberts RS An assessment of clinically useful measures of the consequences of treatment. $\mathrm{N}$ Engl J Med. 1988;318(26):1728-33. https://doi.org/10.1056/ NEJM198806303182605

1. Methods in Epidemiologic, Clinical, and Operations Research-MECOR-program, American Thoracic Society/Asociación Latinoamericana del Tórax, Montevideo, Uruguay. 2. Instituto Nacional de Enfermedades Respiratorias, Ciudad de Mexico, Mexico.

3. Department of Preventive Medicine, Keck School of Medicine, University of Southern California, Los Angeles, CA, USA

4. Divisão de Pneumologia, Instituto do Coração, Hospital das Clínicas, Faculdade de Medicina, Universidade de São Paulo, São Paulo (SP) Brasil.

a. (D) http://orcid.org/0000-0002-1132-5308; b. (D) http://orcid.org/0000-0001-5742-2157; c. (iD) http://orcid.org/0000-0001-6548-1384 